\title{
Wie die Lunge die Lebenskraft bestimmt
}

\author{
9. Dezember 2006, Friedrich-Schiller-Universität Jena
}

\author{
Gast-Herausgeber: C. Uhlemann, Jena
}

Das 8. Symposium zur Ratio und Plausibilität in der Naturheilkunde fand wiederum zum Jahresende in Jena statt. Vertreter der sogenannten Schulmedizin und der klassischen Naturheilverfahren fanden sich zusammen, um zum Thema "Wie die Lunge die Lebenskraft bestimmt» zu referieren.

Das Lungenorgan steht aktuell im Fokus gesundheitspolitischer Diskussionen zum generellen Rauchverbot und den medizinischen, gesundheitsökonomischen und gesellschaftspolitischen Auswirkungen des Rauchens und Passivrauchens. Die Lunge entspricht einem Funktionskreis, der wesentlich für unser Wohlbefinden, unsere Vitalkraft und Vitalität sowie unsere Geisteshelle verantwortlich ist. Nach Prognosen der WHO wird die chronische Bronchitis (COPD), eine der häufigsten Lungenerkrankungen bis 2020 den 3. Platz in der Todesursachenstatistik einnehmen. Dabei «brennt die Lunte nicht nur in der Lunge», sondern ergreift das gesamte System mit Gewichtsverlust, Kachexie, Herz-Kreislauf-Störungen, Muskelschwäche und -atrophie, Osteoporose, Depression und Hormonmangel.

In der griechischen und römischen Antike gab es neben der Schule der Alexandriner, der Empiriker, der Methodiker auch die an die Philosophie der Stoa angelehnte Schule der Pneumatiker, die im ersten vorchristlichen Jahrhundert entstand. Im Pneuma (griech. Lufthauch, Atem Geist) sahen die Pneumatiker, deren theoretische Grundlage aus einer Verbindung von Pneuma und hippokratischer Säftelehre bestand, den Träger des Lebens. "Der Vitalstoff Luft, das Pneuma, wird durch die Lungen in den Körper zur Abkühlung der vom Herzen produzierten Hitze aufgenommen, durchströmt dann zusammen mit dem in der Leber gebildeten Blut den Körper und erhält somit allen Organen der Körperperipherie die Funktion des Organismus." Nach den Pneumatikern ist Gesundheit der Normalzustand des Pneumas, dessen günstigen Tonos (Spannung) man durch Abtasten des Pulses erkennen kann. Auch in der Traditionellen Chinesischen Medizin (TCM) gibt die Qualität des Pulses Auskunft über die körperliche und seelische Verfassung des Menschen Das "Lungen-Qi» ist neben dem "Nieren- und Nahrungs-Qi» eine der 3 elementaren Energiequellen und bestimmt die Vitalität und Geistigkeit eines Menschen.

In der klassischen Naturheilkunde ist die Atmung und damit die Lungenfunktion per se eine der Vogler'schen Grundfunktionen. Eine gesunde Lunge ist mit einer guten Abwehrkraft, einer frohen Gemütslage sowie einem aktiven, motivierten Menschen verbunden. Im Falle von Schwindsucht hielten die alten klassischen Naturheilkundler abhärtende Maßnahmen in Form von Kaltwasseranwendungen, moderatem Bewegen und Luftveränderung (Seereisen) für angezeigt.

Die Lunge als Atmungsorgan im ganzheitlichen bzw. integrativen Sys tem erfährt eine erweiterte Bedeutung, die sich auch in der Metaphorik einer körperbezogenen Sprache ausdrückt: es verschlägt einem den Atem, man kann vor Angst keine Luft holen, es fehlt die Luft zum Atmen, es stockt der Atem, etwas ist atemberaubend schön, jemand hat einen langen Atem oder keine Zeit zum Luftholen. Die Atmung ist die Brücke zwischen Bewusstsein und Unterbewusstsein, durch bestimmte Atemtechniken können tranceähnliche Zustände erreicht werden. Auch muss die Atmung als physiologischer ("struktureller») Vorgang und der Atem ("Odem») als Ergebnis leibseelischer Zustände interpretiert werden. Gesamtheitliche Betrachtungen, wie die Lunge die Lebenskraft bestimmt, wurden auf diesem Symposium erläutert. Zunächst standen Erörterungen zur neuronalen und biochemischen Steuerung der Lungenfunktion im Vordergrund. Hernach wurde aus sog. schulmedizinischer Sicht das Lungenorgan in seiner inneren und äußeren Bedingtheit im Menschen erörtert. Dabei konnte gezeigt werden, dass pneumologische Standardtherapien bei Patienten mit COPD weniger oft effizient waren, wenn jene zugleich depressiv waren. Ein weiteres Thema war die Atmung als Ausdruck der leibseelischen Einheit; Bezüge zu den Stoikern konnten wieder aufgegriffen werden: dass jeder Körper einen Odem, das Pneuma in sich hat, dessen Spannung auf alle Teile wirkt. Es wurden Atemtechniken vorgestellt, die ein Wohlsein auf körperlicher, geistiger und seelischer Ebene erzielen können. Abschließend wurden abhärtende Maßnahmen wie Bewegung, Licht, Luft, Wind und Wasser dargestellt und wie diese die Lungenkraft und damit Vitalkraft jedes Menschen beeinflussen können. Seriell applizierte und adäquat dosierte physikalische Reize (Bewegung, Wasser, Klimafaktoren) bedingen adaptationsphysiologische Mechanismen, die subjektiv spürbar und objektiv in den entsprechenden physiologischen Regelsystemen messbar sind. Somit erfährt der spezifische Lungenfunktionsparameter "Vitalkapazität» im engeren Sinne eine emergente Bedeutung für die Gesamtheitlichkeit eines jeden Menschen im erweiterten Sinn.

Die Medizin der griechischen Antike und anderer Medizinsysteme hat der modernen Wissenschaft zahlreiche Studien und Entdeckungen hinterlassen, die es zu bewahren und für den Menschen als ganzheitliche Persönlichkeit zu nutzen gilt.

C. Uhlemann Jena, Bad Wörishofen

\section{Neuronale und biochemische Steuerung der Lungenfunktion}

PD Dr. med. Andrea Ebersberger

Institut für Physiologie

Friedrich-Schiller-Universität Jena

Die Atmung ist eine der tragenden Säulen unseres Lebens. Der Gasaustausch in der Lunge, der durch die Ventilation der Atemorgane aufrechterhalten wird, bestimmt den Sauerstoffgehalt unseres Blutes, das wiederum die Versorgung aller Gewebe mit Sauerstoff gewährleistet. Sauerstoffminderversorgung hat fatale Folgen, z.B. Schlaganfall oder Herzinfarkt. Die zweite wesentliche Funktion der Atmung ist, das in den Geweben entstehende Kohlendioxid aus dem Körper zu schaffen. Zum einen wird damit in der Lunge die Bindung von Sauerstoff an den roten Blutfarbstoff erleichtert, zum anderen ist $\mathrm{CO}_{2}$ die gasförmige Komponen- 
te des wichtigsten Puffersystems unseres Körpers, d.h. der $\mathrm{CO}_{2}$ des Blutes bestimmt, wie viele Protonen im Blut vorhanden sind, und damit den $\mathrm{pH}$ Wert des Blutes. Dieser wiederum ist wichtig für die Aufrechterhaltung vieler Stoffwechselprozesse z.B. Enzymaktivitäten.

Die Atmung muss sich an den Energieverbrauch in allen unseren Lebens situationen (Hitze, Sport, Schlaf usw.) anpassen. Grundfunktion und Leistungsanpassung der Atmung werden vom Nervensystem gesteuert. Um den Gasaustausch im Gewebe zu regulieren, werden das Ein- und gegebenenfalls das Ausatemvolumen sowie die Atemfrequenz neuronal reguliert. In Ruhelage atmen wir zirka 0,5 1 Luft ein und aus, dabei wird durch Kontraktion des Zwerchfells der Brustraum erweitert, Luft strömt in die Lunge. Wenn das Zwerchfell wieder erschlafft, folgt die Lunge ihrer Eigenelastizität und die passive Ausatmung setzt ein. Soll die Atmung an mehr Körperleistung angepasst werden, benötigen wir auch die Zwischenrippenmuskulatur. Kontraktion der äußeren Zwischenrippenmuskeln hebt den Brustkorb, Kontraktion der inneren Zwischenrippenmuskeln senkt den Brustkorb. Beim bewussten maximalen Ein- und Ausatmen benut zen wir zusätzlich die sog. Atemhilfsmuskeln, die sich über Hals-, Brustund Bauchbereich verteilen. Damit erreichen wir die Ausnutzung der sog Vitalkapazität, die Summe der Volumina, die maximal ein- und ausgeatmet werden können. Auch nach maximaler Ausatmung bleibt Luft in der Lunge zurück (Residualkapazität); zum einen dürfen die Alveolen nicht kollabieren, zum anderen braucht der Gasaustausch Zeit. Währen einer Leistungsanpassung regulieren wir die Atemvolumina innerhalb der Vitalkapazität.

Im Hirnstamm befindet sich das sog. Atemzentrum, bestehend aus eine pontinen, einer dorsalen und einer ventralen Gruppe respiratorischer Neurone; letztere übernimmt die motorische Steuerung der Atemmuskeln. Man findet dort Neurone für die Inspiration, die Zeit unmittelba nach Inspiration (Postinspiration) und die Exspiration. Diese Nervenzel len sind abwechselnd aktiv und geben damit der Einatemmuskulatur einen Rhythmus vor, die passive Ausatmung setzt während der Postinspiration und in der Pause danach ein. Ausatemneurone und damit auch Ausatemmuskeln werden nur bei größeren Leistungsanpassungen aktiv. Der vom Zentralnervensystem vorgegebene Rhythmus wird über afferente Fasern aus der Lunge und anderen Körperbereichen moduliert. Nach dem das Ziel der Atmung die Gewährleistung der $\mathrm{O}_{2}$-Versorgung, die Abgabe von $\mathrm{CO}_{2}$ und die Aufrechterhaltung des optimalen $\mathrm{pH}$-Werts des Blutes ist, wird von peripheren Chemorezeptoren an der Aorta und der Carotis die Blutkonzentration von $\mathrm{O}_{2}, \mathrm{CO}_{2}$ und $\mathrm{H}^{+}$gemessen. Auch im Hirnstamm gibt es Sensoren, sog. zentrale Chemorezeptoren die $\mathrm{CO}_{2}$ und $\mathrm{H}^{+}$messen. $\mathrm{CO}_{2}$ ist bei weitem der wichtigste Atemantrieb, d.h. bei steigenden $\mathrm{CO}_{2}$-Konzentrationen steigt das Atemvolumen pro Minute drastisch an, wobei unter physiologischen Bedingungen hierfür v.a. die zentralen Chemorezeptoren verantwortlich sind. Da die Atemtätigkeit viel Energie kostet, soll sie möglichst ökonomisch erfolgen. Hierfür gibt es Mechanosensoren in den Bronchien. Die dort gemessene Information über die Dehnung der Lunge wird über den Nervus vagus zur ventralen respiratorischen Neuronengruppe im Atemzentrum weitergeleitet und die Einatmung wird beendet. Dieser als Hering-Breuer-Reflex bekannte Vorgang dient auch dem Schutz der Lunge vor Überdehnung. Ganz ähnlich funktioniert die Arbeit von Lungendehnungsrezeptoren in der Muskulatur des Thorax.

Irritationsendigungen im Rachenraum und in den Bronchien können kurzfristig durch chemische oder mechanische Reize erregt werden und führen über den Nucleus tractus solitarii (NTS) zum Atemzentrum un am Ende zum Hustenreflex. Wer beim Tauchen lange die Luft anhält, fühlt sich geradezu gezwungen aufzutauchen und einzuatmen, ein als Deflatations- oder Head-Reflex bezeichneter Vorgang, der ebenfalls übe den NTS im Hirnstamm verläuft. Nicht alle Reflexe sind von Vorteil. Pathologische Situationen, z.B. die Veränderung der Umgebung der Alveolen, entzündliche Schwellungen (erhöhter Extrazellularraum) lösen über die Reizung von schmerzempfindlichen Fasern den juxtaglomerulären Reflex aus, der zur Hemmung der Inspiration führt.

Die bisher genannten Anpassungen der Atmung wurden über sog. spezifische Eingänge zum Atemzentrum eingeleitet. Als unspezifische Eingänge bezeichnete afferente Nervenfasern vermitteln nur indirekte Informationen über den Zustand der Atemgase und des Atemapparats. Muss beispielsweise die Atmung extrem schnell gesteigert werden (100-m-Lauf) ist die Messung der Atemgase zu langsam. In diesem Zeitfenster werden die motorischen Neurone des Atemzentrums über eine Mitinnervation aus der Muskulatur «angetrieben». Auch Hormone (Schwangerschaft), die Körpertemperatur (Fieber), Thermosensoren der Haut (Hitze oder Kälte) sowie Nozizeptoren (Schmerzreize) beeinflussen die respiratorischen Neurone im Sinne einer Leistungsanpassung an die veränderte Gesamtsituation.
Der $\mathrm{O}_{2}$-Gehalt in den einzelnen Organen ist nicht nur von der Atmung abhängig. Der Blutweg ist das Transportsystem für Atemgase, deshalb ist eine Zusammenarbeit zwischen Herz-Kreislauf-System und Atmung nötig. Atmungsneurone der pontinen Gruppe besitzen gemeinsame Interneurone mit kardiovaskulären Neuronen. Die Leistungsanpassung kann dort abgeglichen werden. Es gibt zudem eine atmungsabhängige Sinusarrhythmie am Herzen, die über sympathische Fasern vermittelt ist, d.h. die Arbeit der Schrittmacherzellen des Herzens spiegelt den Atemrhythmus wider. Interessanterweise kann man in vielen Bereichen unseres Körpers kleine atmungsabhängige Fluktuationen in der Aktivität wiederfinden. Muskeltonus, Pupillenweite und Ventilation des Nasenraumes sind nur einige Beispiele. Diese haben gemeinsam, dass sie an die Funktion des vegetativen Nervensystems gekoppelt sind, das seinerseits Einfluss auf die Atmung nimmt. Spätestens hier wird klar, dass das Atemsystem durch seine komplexe Einbindung ins Gesamtsystem Körper weit mehr ist als eine Maschine, die unseren Organismus mit Sauerstoff versorgt.

\section{Literatur}

Jänig W: Integrative Action of the Autonomic Nervous System: Neurobiology of Homeostasis. Cambridge, Cambridge University Press, 2006, pp 420-440.

Thews G, Mutschler E, Vaupel P: Anatomie, Physiologie, Pathophysiologie des Menschen. Stuttgart, Wissenschaftliche Verlagsgesellschaft, 2007.

\section{Mensch und Lunge oder Lunge und Mensch}

\section{Dr. Dr. Claus Kroegel}

Abt. Pneumologie und Allergologie

Friedrich-Schiller-Universität, Jena

\section{Hintergrund und Fragestellung}

Die COPD ist eine Systemerkrankung, die sich neben der Lunge (Emphysem) auch an anderen Organen (Herz, Fettgewebe, Muskulatur, Immunsystem, Knochen) manifestiert. Darüber hinaus besteht eine Assoziation $\mathrm{zu}$ psychischer Morbidität in Form von Depressionen und/oder Angststörungen. So ist die Lebenszeitprävalenz für Depression bei COPD-Patienten mit mehr als $40 \%$ etwa 8-mal höher als die bei der gesunden Normalpopulation. Ebenso übernormal häufig wird eine Angstsymptomatik bei bis zu 50\% und mehr der COPD-Patienten angetroffen $[1,2]$. Allerdings ist bisher unklar, ob für diese Assoziation psychische Störungen als primäre Ursache einer COPD verantwortlich sind oder ob eine bestehende COPD die Entwicklung einer Psychopathie fördert («Henne-Ei-Problem»).

Lunge $\rightarrow$ Mensch: Somatische Ursachen psychischer Störungen bei COPD

Als Ursachen für eine Störung des psychischen Verhaltens durch COPD kommen verschiedene Faktoren in Frage: Nikotinabhängigkeit (bzw. «erzwungene» Nikotinabstinenz), chronische Hypoxämie und Hyperkapnie (respiratorische Insuffizienz), chronische Symptome (z.B. Dyspnoe, Husten, Auswurf), chronische Schlafstörungen (z.B. gestörte Schlafarchitektur), permanent eingeschränkte körperliche Belastbarkeit und Mobilität, soziale Vereinsamung sowie Nebenwirkungen der medikamentöse Dauertherapie. Zusammengenommen handelt es sich um ein Spektrum von Krankheits-assoziierten Faktoren, die eine somatische Ursache der Psychopathie im Rahmen der COPD erklären könnten.

\section{Mensch $\rightarrow$ Lunge: Psychische Ursachen somatischer Störungen bei} COPD

Die Bedeutung der psychischen Komponente (Depression, Angst, emotionale Kapazität) für die Entwicklung der COPD [1, 2] wird von anderen Studien nahegelegt. Depression und Angststörungen beeinflussen den Verlauf der COPD. So kommt es bei depressiven und ängstlichen Patienten zu häufig wiederkehrenden Exazerbationen (bei Depression: 28 vs. 3\%; bei Angststörung: 57 vs. 28\%; bei Depression mit Angststörung: 64 vs. $36 \%$ ). Darüber hinaus beeinflussen emotionale Faktoren die Mortalitätsrate bei COPD. In einer Langzeitstudie [Crocket et al., 2000] an 157 Patienten mit schwerer COPD unter Sauerstoff-Langzeittherapie in den Jahren von 1991 bis 1999 erhöhte eine gesteigerte emotionale Agitiertheit die Mortalität der Patienten. Der Effekt ließ sich allerdings ausschließlich bei Frauen nachweisen. Der Grund hierfür ist nicht bekannt. Möglicherweise geht dieses Ergebnis auf die Tatsache zurück, dass sich bei Frauen mit COPD häufiger Anzeichen für depressive Störungen finden als bei Männern. Aber auch nicht-somatisch erkrankte Frauen leiden häufiger an Depressionen als Männer. Insbesondere für Frauen kann hiernach Angst, Depression und emotionale Agitiertheit als Risikofaktor 
eines vorzeitigen Todes durch die Erkrankung gelten. Schließlich beeinflusst auch die Lebensqualität die COPD-assoziierte Mortalität [Almagro et al., 2002; DeGodoy et al., 2003], wobei die Mortalität mit abnehmender Lebensqualität ansteigt. Umgekehrt verbessert eine Psychotherapie zusätzlich zu physiotherapeutischen Maßnahmen signifikant die körperliche Leistungsfähigkeit und reduzierte gleichzeitig die Mortalität der COPD $[1,2]$

\section{Schlussfolgerungen}

Depressionen und Angststörungen sind bei Patienten mit COPD häufig und Teil der systemischen Krankheitsmanifestation. Die vorhandenen Daten sprechen dafür, dass psychische Faktoren den Verlauf der COPD, vor allem bei Frauen, beeinflussen. Depressionen und Angststörungen be COPD sind mit einer verminderten subjektiven Belastbarkeit, niedrigerem Krankheitswissen und schlechterer Compliance assoziiert. Ob es sich allerdings bei den psychischen Veränderungen um reaktive oder genuine Manifestationen handelt, lässt sich anhand der Ergebnisse nicht entscheiden. Am wahrscheinlichsten ist, dass sich Psyche bzw. Mensch auf der einen Seite und die somatische Krankheitsmanifestation auf der anderen gegenseitig negativ beeinflussen. Unabhängig von der möglichen Beziehung zwischen Mensch/Psyche und Lunge/COPD, deuten die Daten auf die Notwendigkeit einer integrierten Behandlung der somatischen ebenso wie der psychischen Manifestation bei dieser pneumologischen Erkrankung hin.

Literatur

1 Steinkamp G: COPD, die Systemerkrankung: Ernährung: zu wenig beachtet und noch ungelöst. Pneumologie 2003;57:681-689.

-2 Steinkamp G, Dierkesmann R, Gillissen A, Lindner M, Mitfessel H, Morr H, Pfeifer M, Schultze-Werninghaus G, Sybrecht G, Worth H: COPD und Psyche ein Überblick. Pneumologie 2005;59:819-830.

\section{Der Atem - Kennzeichen der leibseelischen Einheit}

PD Dr. med. Dominik Irnich

Interdisziplinäre Schmerzambulanz

Klinikum der Universität München

Aus physiologischer Sicht bezeichnet der Atem die Luftmenge, die bei der Tätigkeit des Atmens (Luftholen oder Atemzug) bewegt wird (Einatmen $=$ Lunge mit Luft füllen, Ausatmen $=$ Luft ausstoßen $)$. Der Vorgang des Atmens ist die Atmung. Dabei unterscheidet die Humanmedi zin die äußere Atmung (Lungenatmung) und die innere Atmung (Zellatmung). Als innere Atmung bezeichnet man den Gasaustausch zwischen Blutkreislauf und Lungenalveole, also die Aufnahme von $\mathrm{O}_{2}$ aus dem Blut in die Körperzellen und die Abgabe von $\mathrm{CO}_{2}$ in das Blut. Der Be griff der äußeren Atmung umschreibt den Vorgang der Exspiration und Inspiration.

Betrachten wir aber den Begriff des Atems aus kultur- und medizinhistorischer Sicht ergibt sich ein deutlich erweitertes, den gesamten Menschen umfassendes Bild. So ist der Atem in der griechischen Heilkunst der Sitz der Seele im Zwerchfell. Geist, Sinn und Zwerchfell bekommen den gleichen Namen. Diogenes von Apollonia, Naturphilosoph und angesehener Arzt im antiken Griechenland (500 v. Chr.) wird zitiert mit den Worten «Das Leben und das Denken in allen lebendigen Wesen ist bewirkt durch die Luft, welche sie einatmen, und an diesen Stoff geknüpft.» Auch Hippokrates verleiht Luft und Atem eine besondere Bedeutung: «... den Verstand aber verleiht ihm die Luft. Denn ein jeder Körperteil besitzt soviel Verstand, als er an der Luft Anteil hat.»

In den folgenden Jahrhunderten entwickelte sich die Ärzteschule der Pneumatiker, die das in der Atemluft gegebene Prinzip (Pneuma) als Träger des Lebens und sein Versagen als Grundlage von Krankheiten definierten. Hauptvertreter, dieser dynamisch-vitalistischen Theorie waren Athenaios und von Archigenes. Die Sichtweisen der Pneumatiker fanden sich im spätantiken Gnostizismus oder der Hermeneutik wieder. Der immaterialistische Geist ist verantwortlich für die Vorgänge im Universum und damit auch im Menschen. Das Verständnis kann wiederum mit dem menschlichen Geist erschlossen werden. «Pneuma» kann also als Geist, Kraft, Hauch oder Atem übersetzt werden. Die Lunge, als Organ mit be sonderem Bezug zum Atem, und die Lungenfunktion bekommen damit eine zentrale Stelle in Diagnostik und Therapie von Krankheitszuständen. Folgt man dieser Linie ergibt sich der Bezug zum Begriff des Leibes, der in der westlichen Kulturgeschichte einen engen Bezug zum Leben bekommt (althochdtsch.: līb = Leben; mittelhochdtsch.: līp/līb = Leben, Körper; angelsächs.: līf = Leben; altnord.: līf = Leben). Im Gegensatz zu
Das Funktionsbild Lunge

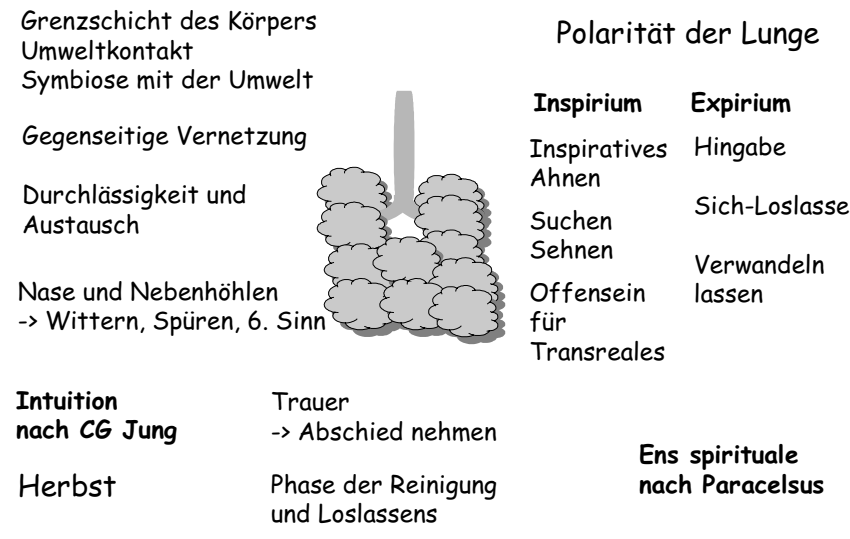

Abb. 1. Funktionsbild Lunge nach TCM, erweitert um medizinhistorische und psychosomatische Aspekte, modifiziert nach Gleditsch [1].

einem mechanistischen, materialistischen Funktionsbild der Lunge bekommt also die Lunge im erweiterten Sinn eine tragende Bedeutung in der leibseelischen Einheit des Menschen.

Dieses Bild zieht sich auch durch die Literatur der letzen Jahrhunderte: Immer wieder wird der Atem mit seelischem Wohlbefinden in $\mathrm{Zu}$ sammenhang gebracht (z.B. Honoré de Balzac «Erfrische Deine Seele mit dem Atem der Morgenröte»). Dieser Aspekt findet sich auch in den östlichen Kulturkreisen seit Anbeginn. Sei es Buddha («So Du zerstreut bist, lerne auf den Atem achten»), Bhagavad-Gita («Als Lebenswärme werd ich offenbar - in Wesen welche atmen. Zweifach ist mein Atem, innerlich und äußerlich-geistig und physisch. So ernähre ich alles.») oder andere überlieferte indische Weisheiten («Der Atem ist das Tor zur Wirklichkeit»).

Auch in der Traditionellen Chinesischen Medizin (TCM) wird ein umfassendes Funktionsbild der Lunge gegeben. So nimmt die Lunge das AtemOi (Oi als Lebensenergie) auf und verteilt es im Körper. Sie bildet als Schleimhautorgan die Grenzschicht zur Umwelt und wehrt bei guter Funktion die schädigenden Einflüsse von außen ab. Das Standardwerk der TCM, das Huangdi Neijing Suwen, beschreibt die Lunge «als Amtssitz der hohen Minister, in der Ordnung und Einteilung entspringen». Fasst man die Sichtweisen der verschiedenen Kulturen und Denkrichtungen zusammen, so ergibt sich ein umfassendes Funktionsbild «Lunge» (Abb. 1), welches über die physiologische Funktion hinaus phänomenologisch Aspekte des Atems zusammenträgt [1]. Dabei wird das Menschsein in seiner seelisch-geistigen Dimension erfasst. Dem Atem in seiner umfassenden Sichtweise wird auch Rechnung getragen in den verschiedenen psychophysischen Atemtherapien (z.B. nach Middendorf), in Verfahren, welche auf die Körperwahrnehmung besonderen Wert legen (z.B. Feldenkrais, Psychotonik) und in verschiedenen östlichen Methoden wie Yoga, Qigong und Tai Chi. Die Atemerfahrungen umfassen dabei den Atemrhythmus, die Körperraumerfahrung, die Erfahrung von Begrenzung und Entgrenzung, das Erkennen des zeitlichen Ablaufes, das Spüren der Intensität des Atems, das Bewusstwerden der Kraft der Atmung und die Rückkoppelung des Atemflusses.

Auch in der modernen Medizin werden Zusammenhänge zwischen der physiologischen Funktion der Lunge und der menschlichen Psychosomatik zunehmend anerkannt. So beschreibt die Psychosomatik das Auftreten einer Seufzeratmung (unwillkürlich vertiefte Atemzüge) bei einer als schwer empfundenen Last oder Leistung. Die verhaltene Atmung (oft das Gefühl, nicht richtig durchatmen zu können, Atmungskorsett) tritt bei zurückhaltenden und gehemmten Patienten mit einer Tendenz des sich Verschließens auf. Das nervöse Hüsteln wird eher einem aggressiven Charakter zugeordnet. Beim Hyperventilationssyndrom können neben organischen Ursachen (Lungenerkrankung, Erregbarkeitssteigerungen oder tetanische Bereitschaft) auch psychische Faktoren an der Entstehung beteiligt sein; dazu gehören heftige Emotionen oder Ich-schwache Persönlichkeiten mit abhängigem Verhalten. Selbst beim Asthma bronchiale beobachtet die Psychosomatik ein ambivalentes Bindungsverhalten zur Mutter bei konfliktvermeidendem Vater. Eine Rolle kann dabei aus psychosomatischer Sicht auch die unterdrückte Aggressivität spielen. Der 
Patient kann seinem Ärger nicht Luft machen. Als auslösend für Lungenerkrankungen werden oft Konflikte im Bereich Trennung oder Anteilnahme angesehen. Somit scheinen Trauer und Aggression einen besonderen Bezug zur Lungenfunktion haben. Diese Erfahrung findet sich auch in den Überlieferungen der TCM, die dem Funktionskreis Lunge die Trauer zuordnet. Die Aggression scheint für die Lungenfunktion eine besondere Bedeutung zu haben. Der Zusammenhang von Lungenfunktion und Aggression wird eindrucksvoll von einer aktuellen wissenschaftlichen Unter suchung beschrieben: An einem Kollektiv von 670 Personen, bei denen die Lungenfunktion mit emotionalen Parametern in Zusammenhang ge bracht wurde, zeigte sich, dass pro Punkt auf der 50 Punkte-Wut-Skala (Cook-Medley Hostility Scale) die Einsekundenkapazität (FEV1) der Lunge um durchschnittlich $9 \mathrm{ml}$ pro Jahr abnimmt [2]

Aktuelle Studien beschreiben aber nicht nur, dass Emotionen Einfluss au die Lungenfunktion haben, sondern es ist darüber hinaus die geistige Grundhaltung des Menschen, die einen engen Bezug zur Gesundheit besitzt. So konnte bereits 1999 an einem Kollektiv von über 35000 Personen gezeigt werden, dass die spirituelle Ausrichtung der wichtigste Gesundheitsfaktor ist [3]. Auch bezogen auf die Lungengesundheit ist die spirituelle Festigkeit ein protektiver Faktor [4]

Welche Schlussfolgerungen lassen sich aus diesen Gedanken, Aphorismen, Erfahrungen, Überlieferungen und wissenschaftlichen Erkenntnissen ziehen? Der bewusste Atem und das Training desselben auf leibliche und seelischer Ebene tragen zu Gesundheit und Wohlbefinden bei. Der Arzt sollte deshalb bei Erkrankungen seines Patienten diesen Aspekt in die Diagnostik und Therapie einbeziehen, um so zu einem umfassenden, ganzheitlichen Krankheitsverständnis zu kommen. Therapeutisch gilt es dann, neben der Anwendung der bekannten und bewährten Therapiekonzepte, gemeinsam mit dem Patienten einen Weg der Rückkehr zur leibseelischen Einheit zu finden. Der Atem kann dabei eine entscheidende Rolle spielen.

\section{Literatur}

1 Gleditsch J: Mikro-Aku-Punkt-Systeme. Grundlagen und Praxis der somatotopischen Therapie. Hippokrates, 2002, p 243.

2 Kubzansky LD et al: Angry breathing: A prospective study of hostility and lung function in the Normative Aging Study. Thorax 2006;61:863-8.

3 Grossarth-Maticek R: Systemische Epidemiologie und präventive Verhaltensmedizin chronischer Erkrankungen. Strategien zur Aufrechterhaltungen der Gesundheit. Berlin, de Gruyter, 1999

4 Maselko et al.: Religious service attendance and decline in pulmonary function in a high-functioning elderly cohort. Ann Behav Med 2006;32:245-53.

\section{Bewegung - formativer Reiz für die Lungenkraft}

Univ.-Prof. Dr. med. Holger Gabriel

Institut für Sportwissenschaft

Friedrich-Schiller-Universität Jena

Der positive Einfluss eines körperlich aktiven Lebensstils auf die Lebenserwartung wird im Mittel mit 5 Jahren beziffert; dabei ist von einem wöchentlichen Energieverbrauch von 1200-2000 kcal durch gezielte körper liche Aktivität auszugehen. Der Idealfall ist die Integration in die sinnvolle Alltagsgestaltung unter Berücksichtigung einer Mindestdauer einzelner Belastungseinheiten von mindestens $30 \mathrm{~min}$, einer Mindestintensität von $50 \%$ der maximalen Leistungsfähigkeit und dem dynamischen Einsatz möglichst großer Muskelgruppen. Die WHO und der Weltsportärzteverband haben daher 1994 alle Regierungen der Erde aufgefordert, folgende Ziele bevorzugt zu verfolgen [5]: «Tägliche körperliche Aktivität sollte den Eckpfeiler eines gesunden Lebensstils darstellen; Kindern und Jugendlichen sollten Gelegenheiten für tägliche Bewegungsprogramme geboten werden mit dem erzieherischen Effekt, dass körperliche Aktivität sich zu einer lebenslangen Gewohnheit entwickeln sollte; Erwachsene sollten eine täglich mindestens 30-minütige gezielte körperliche Aktivität von mäßiger Intensität auf sich nehmen, z.B. schnelles Gehen, Wandern oder Treppensteigen. Intensivere Belastungen wie langsamer Dauer lauf, Radfahren und Schwimmen könnten zusätzliche gesundheitliche Vorteile erbringen; Frauen benötigen spezifische Möglichkeiten zur Aus übung von körperlichem Training; Ältere und alte Personen, deren Prozentsatz in der Bevölkerung weltweit ansteigt, sollten ermutigt werden, einen aktiven Lebensstil unabhängig vom Alter beizubehalten, um so ihre körperliche Unabhängigkeit zu bewahren und alternsbedingten körperlichen und geistigen Leistungsverlusten entgegenzuwirken; Behinderte
Personen und chronisch Kranke sollten in besonderer Weise bei der Erarbeitung von Möglichkeiten zu gezielter körperlicher Aktivität unterstützt werden.»

Die sog. «Low-level-Inflammation» spielt bei der Entstehung der Arteriosklerose und ihrer Folgeerkrankungen eine wesentliche Rolle. Körperliche Aktivität induziert antiinflammatorische Mechanismen, die die proinflammatorische Last, welche z.B. durch Infektionen, Übergewicht, Rauchen und hohe Fettzufuhr in der Ernährung entsteht, wirkungsvoll reduzieren kann [1]. Voraussetzung für die antiinflammatorische Wirkung körperlicher Aktivität ist ein Training der allgemeinen aeroben Muskelausdauer. Andere Formen des Muskelausdauertrainings wie das lokale oder das allgemeine anaerobe Muskelausdauertraining können nach derzeitigen Vorstellungen keine allgemeinen antiinflammatorischen Mechanismen induzieren, wirken also nicht präventiv Arteriosklerosefolgeerkrankungen entgegen. Körperliches Training führt zu morphologischen und funktionellen Adaptationen. Dabei sind die Trainingsprinzipien zu beachten. Eine angemessene Dauer, Intensität und Schwierigkeit von Trainingseinheiten und -zyklen ist erforderlich. Ebenso ist deren Adaptation an das Alter, den Trainingszustand und eventuell Erkrankungen zu beachten. Eine der Beanspruchung angemessene Regenerationszeit nach Belastungen ist Voraussetzung für einen sinnvollen Trainingsprozess bei Gesunden und Kranken. Werden diese Punkte beachtet, führt körperliches Training u.a. zur Anpassung von Muskeldicke, -kraft und -ausdauer der trainierten Muskulatur, des Bau- und Energiestoffwechsels, des Immunsystems, innerer Organe wie dem Herz und Organen des Halte-/Bewegungs-/Stützsystems.

Bewegung und körperliche Aktivität sind Muskelarbeit, die in einer bestimmten Zeit geleistet werden. Diese durch die Muskulatur erbrachte Leistung bedarf der Oxidation von Energie der liefernden Nährstoffe Kohlenhydrate, Fette und - in beschränktem Ausmaß - auch Eiweiß. Dadurch kann der Muskulatur Adenosintriphosphat (ATP) als Energieträger in der erforderlichen Zeit zur Verfügung gestellt werden. Abhängig von der Art der zur Verfügung stehenden Nährstoffe wird unterschiedlich viel Sauerstoff benötigt, der über die Lunge aufgenommen und über das Blut zur Muskulatur transportiert wird. Physiologisch steht die Sauerstoffaufnahme mit der muskulären Leistung in engem Zusammenhang, so dass Leistung im physiologischen Sinn letztendlich $\mathrm{O}_{2}$-Aufnahme in den Organismus bedeutet. Bestimmte Faktoren und Umstände können die $\mathrm{O}_{2}$-Aufnahme und damit die körperliche Leistungsfähigkeit vermindern. Ohne Anspruch auf Vollständigkeit sind diese auf muskulärer Ebene Bewegungsmangel, Übergewicht und neuromuskuläre Erkrankungen, auf Ebene des Bluts und des Bluttransports Erkrankungen des Herz-Kreislauf-Systems, die mit Zirkulationseinschränkungen einhergehen, Blutbildungsstörungen und $\mathrm{O}_{2}$-Bindungsstörungen in den roten Blutkörperchen und auf Lungenebene obstruktive und restriktive Lungenerkrankungen sowie Atemregulationsstörungen. Hinzu kommen Diffusionsstörungen zwischen den Kompartimenten und äußere Umstände, die mit der Verminderung der $\mathrm{O}_{2}$-Konzentration und dem $\mathrm{O}_{2}$-Partialdruck einhergehen. Die höchsten $\mathrm{O}_{2}$-Aufnahmen werden mit Hilfe der Spiroergometrie bei Maximaltests von 10-12 min Dauer unter Einsatz möglichst großer Muskelgruppen erreicht, die auch durch ein allgemeines Ausdauertraining langjährig trainiert wurden. Dabei finden sich Werte von etwa $90 \mathrm{ml} / \mathrm{min} / \mathrm{kg}$ bei Radrennfahrern, Marathonläufern und Skilangläufern. Hierbei handelt sich um Athleten im 3. oder 4. Lebensjahrzehnt. Kranke und alte Menschen haben eine maximale $\mathrm{O}_{2}$-Aufnahme von weniger als etwa $20 \mathrm{ml} / \mathrm{min} / \mathrm{kg}$. Ein Training der allgemeinen aeroben Muskelausdauer durch Gehen, Walking, Jogging, Laufen, Radfahren, Rudern, Skilanglauf etc. führt bei Beachtung der grundlegenden Trainingsprinzipien zu einem Anstieg der Sauerstoffaufnahme und damit der allgemeinen körperlichen Leistungsfähigkeit. Kann aus Erkrankungsgründen kein Training der allgemeinen aeroben Muskelausdauer durchgeführt werden, ist eine Verbesserung der Lungenfunktion, auch eine morphologische Anpassung der Atemmuskulatur durch ein lokales Training der Muskelausdauer möglich. Die allgemeine Muskelausdauer verbessert sich dadurch jedoch nicht. Lokales Training der Muskelausdauer wie beispielsweise durch ein inspiratorisches Muskeltraining führt $\mathrm{zu}$ einer Verminderung der lokalen Muskelermüdung, von Atemgasparametern und Lungenfunktionsparametern, also einer Zunahme der «Lungenkraft»; die Verbesserung der maximalen $\mathrm{O}_{2}$-Aufnahme wird dadurch nicht erreicht. Somit können Patienten mit restriktiven und obstruktiven Lungenerkrankungen, die ein solches therapeutisches Muskeltraining durchführen, positive lokale Effekte mit entsprechenden positiven Auswirkungen auf die allgemeine Leistungsfähigkeit erwarten. Im Vergleich zu Steigerungen der maximalen $\mathrm{O}_{2}$-Aufnahme durch ein Training der allgemeinen aeroben Muskelausdauer bleiben diese mit Bezug auf die positiven Auswirkungen im alltäglichen Leben deutlich zurück [2-4]. 
Fazit: Körperliche Aktivität trägt zur Prävention von Krankheiten innerer Organe und des Halte-/Bewegungs-/Stützsystems bei und erhöht die durchschnittliche Lebenserwartung um etwa 5 Jahre. Unter Beachtung der allgemeinen Trainingsprinzipien wirkt das Training der allgemeinen aeroben Muskelausdauer antiinflammatorisch und antiatherogen. Dazu ist an den meisten Tagen der Woche ein dynamischer Einsatz möglichs großer Muskelgruppen erforderlich. Das Training der lokalen Muskelausdauer kann zu einer Adaptation der beanspruchten Muskulatur führen. Auch sehr niedrig leistungsfähige Menschen können durch ein Training der Muskelausdauer profitieren. Soweit es die Grunderkrankung ermöglicht, ist ein Training der allgemeinen aeroben Muskelausdauer erstrebenswert, womit insbesondere die Belastbarkeit im Alltag spürbar verbessert werden kann.

Literatur

1 Bruunsgaard H: Physical activity and modulation of systemic low-level inflammation. J Leukocyte Biol 2005;78:819-35.

2 Freeman W, Stableforth DE, Cayton RM, Morgan MDL: Endurance exercise capacity in adults with cystic fibrosis. Respir Med 1993;87:541-9.

- 3 Geddes EL, Reid WD, Crowe J, O'Brien K, Brooks D: Inspiratory muscle training in adults with chronic obstructive pulmonary disease: a systematic review. Respir Med 2005;99:1440-58.

4 Gimenez M, Servera E, Vergara P, Bach JR, Polu JM: Endurance training in patients with chronic obstructive pulmonary disease: a comparison of high versus moderate intensity. Arch Phys Med Rehabil 2001;81:102-9.

5 Kölner Deklaration «Gesundheit und körperliche Aktivität - Erklärung der Weltgesundheitsorganisation und Weltsportärzteverband». Dtsch Z Sportmed 1994;45:170

\section{Licht, Luft und Wind - elementare therapeutische Säulen}

\section{Prof. Dr. Dr. Angela Schuh}

Institut für Gesundheits- und Rehabilitationswissenschaften Universität München

Klimatherapie gehört zu den Naturheilverfahren und beschäftigt sich mit den gesundheitsfördernden Auswirkungen des Wetters und des Klimas auf den Menschen sowie deren Einsatz und Nutzen zur Prävention, Therapie und Rehabilitation. Insbesondere chronische Erkrankungen von Haut und Atemwegen sind eine Domäne der Klimatherapie. Sie wird auch bei Osteoporose, Herz-Kreislauf-Erkrankungen, Trainingsmangel des ganzen Körpers, jahreszeitlich abhängigen Verstimmungen und saisonaler Depression sowie zur Rekonvaleszenz nach schweren Erkrankungen eingesetzt [1]. Klimatherapie hat einen besonderen Stellenwert in der Sekundärprävention und allgemeinen Gesundheitsförderung. Heute wird Klimatherapie an Nord- und Ostsee vor allem als Rehabilitationsmaßnahme bei chronischen Erkrankungen und in Form von Mutter-Kind-Kuren in Fachkliniken durchgeführt. Im Hochgebirge und in den Mittelgebirgen wird Klimatherapie ebenfalls stationär und als ambulante indikationsbezogene Kurorttherapie vorgenommen. Dabei werden die Klimaexposi tionsverfahren (Terraintherapie, Frischluft-Liegetherapie und Heliotherapie) sich gegenseitig unterstützend kombiniert und gegebenenfalls durch thalassotherapeutische, sporttherapeutische, balneologische und weitere physikalisch-therapeutische Maßnahmen ergänzt. Angeboten wird Klimatherapie in den Heilklimatischen Kurorten, Seebädern und Seeheilbädern Deutschlands und in verschiedenen anderen europäischen Ländern.

Bei Patienten mit Erkrankungen der Atemwege sind hohe Luftreinhei und die Absenz bzw. Reduktion von Pollen und weiteren Aeroallergen das wesentliche Fundament der Klimatherapie. Die Entlastung der Atemwege ist dabei das primäre Ziel der Therapie. Dies gilt insbesondere fü das extrinsische und das gemischte Asthma. Vor allem bei Asthma im Kindesalter stellen Pollen zu 80\%, Hausstaubmilben zu 60-80\% und Schimmelpilze zu 20\% die relevanten Allergene dar. Da sich Allergien meist erst im Laufe der Zeit durch Akkumulation, d.h. häufige Auseinandersetzung mit einem Allergen manifestieren, lässt sich z.B. Heuschnupfen durch regelmäßigen Aufenthalt in pollenfreier Umgebung während der Hauptblütezeit vollkommen vermeiden und ein eventueller Abstieg in unteren Atemwege sowie die Manifestation eines allergischen Asthma bronchiale verhindern. Es wurde darüber hinaus gezeigt, das Luftschadstoffe nicht nur durch Modifizierung des Epithels einen direkten Einfluss auf das Immunsystem haben, sondern auch eine Zunahme der Allergenität der relevanten Aeroallergene hervorrufen [2]. Durch Interaktion von Pollen und Luftschadstoffen kommt es zu einer vermehr- ten bzw. veränderten Pollenfreisetzung. Zusätzlich werden während des Pollenwachstums und Heranreifens der Pollen Schwermetalle, Schwefelbestandteile und weitere Schadstoffe aufgenommen. Somit ergänzen sich Luftschadstoffe und Pollen nicht nur additiv, sondern potenzieren die allergische Reaktion. Hohe Luftreinheit stellt dabei nicht nur für Asthma bronchiale, sondern auch für alle weiteren, auch nicht allergischen Atemwegserkrankungen wie COPD ein wesentliches klimatherapeutisches Element dar. Darüber hinaus rufen vor allem kühle, frische (nicht kalte!) Luft und Wind günstige Adaptationen an den Atemwegen hervor. Das therapeutische Ziel der Abhärtung besteht aus einer unmittelbaren gesundheitsschonenden Reaktion des Körpers auf einen Kaltreiz und aus gesundheitsfördernden Veränderungen im Körper, die unter anderem eine erhöhte Abwehr gegen Infekte nach sich ziehen. So nimmt nachgewiesenermaßen die Durchblutung der Schleimhäute der Atemwege nach klimatherapeutischer Abhärtung zu. Die systematische Anwendung leichter Kältereize ist Grundlage für klimatherapeutische Anwendungen am Meer und im Gebirge.

In Deutschland gibt es drei bioklimatische Zonen, in denen Klimatherapie durchgeführt wird: Das Mittelgebirgsklima, das Hochgebirgsklima und das Seeklima. Die klimatischen Bedingungen der Mittelgebirge weisen einen überwiegend schonenden und entlastenden Einfluss auf, die reizintensiven Elemente sind im Vergleich $\mathrm{zu}$ den anderen Klimazonen deutlich geringer. Für die Atemwege ist vor allem bedeutsam, dass die ausgedehnten Waldgebiete der Mittelgebirge ein großes Reinluftreservoir darstellen, sie verbrauchen $\mathrm{CO}_{2}$ und produzieren $\mathrm{O}_{2}$. Die Blätter und Nadeln der Bäume filtern bzw. «kämmen» anthropogene Schadstoffe wie gasförmige Beimengungen, Ruß- und Staubteilchen aus der Luft heraus. Aufgrund des Schonklimas und der Entlastung von Luftschadstoffen ist eine Klimatherapie im Mittelgebirge vor allem bei Atemwegserkrankungen mit sehr schwerer Ausprägung (z.B. ausgeprägte COPD, Emphysem) indiziert. Bei allergischen Atemwegserkrankungen ist sie allerdings kontraindiziert. Allergische Reaktionen des Atemtrakts und weitere Atopien wie Neurodermitis werden im Hochgebirgsklima zwischen 1000 und $2500 \mathrm{~m}$ erfolgreich behandelt. Auch nicht allergische Atemwegserkrankungen wie chronische Bronchitis werden im Hochgebirgsklima verbessert. Die wirksamen Faktoren sind der verringerte Sauerstoffpartialdruck und die reduzierte Lufttemperatur. Das reizintensive Hochgebirgsklima zeichnet sich aber auch durch hohe Luftreinheit und Allergenreduktion aus, wobei besonders hervorzuheben ist, dass ab zirka 1200 m Hausstaubmilben, deren Exkremente die wichtigsten Aeroallergene beim allergischen Asthma darstellen, nicht mehr lebensfähig sind In diesen Höhenlagen gibt es auch keine Schimmelpilze, und zahlreiche Pollenarten finden sich gar nicht oder blühen zum Flachland zeitlich verschoben

An Nord- und Ostsee üben vor allem die Kältereize aufgrund der niedrigen Lufttemperaturen und des intensiven und turbulenten Windes klimatherapeutische Reize aus. Diese stellen hocheffektive Präventivmaßnahmen gegen Erkältungskrankheiten bzw. eine gesteigerte Infektanfälligkeit der oberen Atemwege dar. Die Luftreinheit an der See sorgt aber auch für eine konkrete Entlastung. Bei Patienten mit Erkrankungen der Atemwege bewirkt außerdem die feuchte Luft an der See, dass die Austrocknung der Schleimhäute der Atemwege während des Atmens vermindert wird. Durch die Abkühlung der Haut während der Abhärtung kommt es außerdem zu einer Reduktion der Temperatur der Schleimhäute, so dass dort der Atem kondensiert. Dadurch entsteht eine zusätzliche Befeuchtung. Durch das Einatmen des maritimen Aerosols beim Aufenthalt in der Brandungszone bzw. am Strand, wobei die kleinen Salzpartikel bis in die Bronchien vordringen können, kommt es zur Sekretolyse, der Schleim wird dünnflüssiger. Durch die verbesserte Zilienmobilität in den Bronchien wird der Abtransport aus der Lunge verbessert. Für die Behandlung von Erkrankungen, die allergisch bedingt sind oder eine allergische Disposition aufweisen, steht zusätzlich die Entlastung der Atemwege durch den Aufenthalt in einem Klima, das im günstigsten Fall frei von Allergenen ist, im Vordergrund der klimatherapeutischen Strategie. Das Seeklima bietet allerdings nur für Pollenallergiker Entlastung, da an Nord- und Ostsee wegen der immer herrschenden hohen Luftfeuchtigkeit Hausstaubmilben und Schimmelpilze vorhanden sind.

Die Erfolge der Klimatherapie sind zu einem großen Teil anhand sog. «harter» Parameter dokumentiert, wobei die Verbesserungen des jeweiligen Krankheitsbildes zunehmend nach den Kriterien der sog. evidenzbasierten Medizin abgesichert werden. Neuere Studien erfassten dabei z.B. an der See und im Hochgebirge die längerfristige Wirkung der Klimatherapie auf Atopien, Haut- und Atemwegserkrankungen. So zeigte sich, dass sich die klinischen Parameter bei Atopikern durch den Höhenaufenthalt schnell und deutlich verbessern und die Effekte auch langfristig anhalten können. Eine kontrollierte Studie bestätigt den hervorragenden 
und längerfristig anhaltenden Effekt der Hochgebirgsklimatherapie bei Jugendlichen mit Asthma bronchiale [3]. Die untersuchten Patienten hat ten eine Hausstaubmilbenallergie, wurden mit Kortikosterioden behandelt und unterzogen sich einer 10-wöchigen Kur in 1600 m. Die Ergebnisse zeigen eine signifikante Verbesserung aller gemessenen Parameter im Vergleich zu einer auf Seehöhe behandelten Kontrollgruppe. Die 10wöchige Allergenvermeidung resultierte in einer sichtbaren Zunahme de Lebensqualität und Verbesserung der bronchialen Reaktion, die Histamin- und AMP-Konzentrationen konnten im Provokationstest währen des Höhenaufenthaltes verdoppelt werden. Begleitet wurde die Verbesserung von einer Abnahme der Eosinophilen und der Entzündungsparameter. Diese signifikante Verbesserung des Krankheitsbildes ebenso wie die Steigerung der Lebensqualität war noch 6 Wochen nach Beendigung des Höhenaufenthaltes festzustellen, während sich bei der Kontrollgruppe keine Veränderung der Parameter ergab. Die Ergebnisse sind bei sons unverändertem Therapieregime allein der Haustaubmilbenfreiheit in dieser Höhenlage zuzuschreiben und zeigen eindeutig, dass eine zeitlich begrenzte Allergenvermeidung im Hochgebirgsklima einen klinischen Benefit bei atopischen Jugendlichen mit leichtem bis mittelschwerem Asthma bronchiale hat. Die Aktivität von Mast- und T-Zellen, die neben den Lymphozyten die hauptsächlichen entzündlichen Infiltrate in den Schleimhäuten der Atemwege von Asthmapatienten darstellen, wird durch die Absenz von Hausstaubmilben in der Höhe abgeschwächt, was z.B. zu einer reduzierten Sekretion von Zytokinen und Eosinophilen führt; selbst die Abschwächung der Entzündung wird durch die Allergenvermeidung allein hervorgerufen. Diese Effekte halten auch nach Rückkehr und erneuter Auseinandersetzung mit dem Allergen noch längerfristig an. Die Langzeiterfolge werden auch durch mehrere repräsentative Erhebungen unterstrichen, die eine signifikante Reduktion der Fehlzeiten von Patienten mit Asthma bronchiale nachweisen. Auch bei weiteren Indikationen der Klimatherapie wie Psoriasis, Neurodermitis, Osteoporose, Koronare Herzkrankheit, metabolischem Syndrom oder sog. «funktionellen» Störungen des Herz-Kreislauf-Systems ohne Organbefund sprechen Studienergebnisse für eine rasch einsetzende und anhaltende Reduzierung der Symptome.

Die moderne Klimatherapie wird somit heute auf wissenschaftlich gesi cherter Grundlage zur gezielten Behandlung fest umrissener Krankheitsbilder und chronischer Leiden sowie in der Prävention bzw. Gesundheitsförderung mit großem Erfolg und langfristiger Besserung der Beschwerden eingesetzt.

\section{Literatur}

1 Schuh A: Klima- und Thalassotherapie. Hippokrates, 2004.

2 Behrendt H, Krämer U, Schäfer T, Kasche A, Eberlein-König B, Darsow U, Ring $\mathrm{J}$ : Allergotoxicology - a research concept to study the role of environmental pollutants in allergy. ACI Intern 2001;13:122-8.

3 Grootenhorst DC, Dahlen SE, Van Den Bos JW., Duiverman EJ, Veselic-Charvat $\mathrm{V}$ et al: Benefits of high altitude allergen avoidance in atopic adolescents with moderate to severe asthma, over and above treatment with high dose inhaled steroids. Clin Exp Allergy 2001:31:400-8.

\section{Kneipp'scher Oberguss - Schutz für den Bronchitiker?}

Dr. med. Katrin Goedsche, Institut für Physiotherapie,

Prof. Dr. med. Christine Uhlemann,

Kompetenzzentrum Naturheilverfahren, Klinik für Innere Medizin II, Friedrich-Schiller-Universität Jena

Aufgrund jahrelanger Beobachtungen wurden bereits im vorigen Jahrhundert Kaltwasser-Anwendungen durch Pfarrer S. Kneipp als «abhär tend» für den Organismus erkannt. Die drei wichtigsten Aufgaben des Wassers waren für ihn «Auflösen, Ausleiten und Kräftigen». Auch der Bauer V. Prießnitz (1799-1855) erwähnte die heilende Kraft des Wassers, indem dies den Organismus stärkt und die Selbstheilungskräfte anregt Unter «Abhärtung» wird heute eine durch funktionelle Adaptation (Konditionierung) entstandene Stärkung des Organismus gegenüber exogenen und endogenen noxischen Reizen verstanden. Dadurch kann z.B. die Häufigkeit von Infekten der oberen Luftwege abnehmen. Eine Konditionierung des Organismus (in diesem Falle des Immunsystems) kann durch verschiedene physikalische Reize erfolgen: z.B. durch moderaten Sport (kinetischer Reiz), UV-Licht (klimatischer Reiz), kaltes Wasser (thermischer Reiz). Prinzipiell ist die adäquate Dosis des seriell applizierten Reizes zur gewünschten Störung der Homöostase und damit adaptiven $\mathrm{Be}-$ einflussung der physiologischen Regelsysteme von Bedeutung.
Studien zur immunologischen Reaktion auf Kneipp'sche Hydrotherapie zeigen, dass nach Kaltwasseranwendungen eine Modulation der spezifischen Immunabwehr in Richtung der zellvermittelten Typ1-Reaktion erfolgte. Dies war verbunden mit einem Anstieg der Leitzytokine IL-2 und IFN- $\gamma$ sowie einer Abnahme von Infektionskrankheiten. Im Rahmen einer ganzheitlichen Therapie bei Patienten mit chronisch obstruktiver Bronchitis (COPD) dienen Kneipp'sche Obergüsse der Stärkung der Immunabwehr. Dadurch könnten die den Krankheitsverlauf negativ beeinflussenden Exazerbationen mit Infekten der oberen Luftwege verringert werden. An die COPD-Stadien (nach GOLD-Stufenschema 2003) adaptiert ist dies jedoch nur bis zum Stadium II in Form von kalten Güssen möglich. Die Stadien III-IV sind aufgrund der fortgeschrittenen chronischen Entzündung nur noch milden Reizen aussetzbar (z.B. «kleine» Wasseranwendungen, temperiertes Wasser). Die Reaktionsfähigkeit des Organismus und die Möglichkeit der Selbstheilung sind in diesen Stadien reduziert.

Eine prospektive, klinische Pilotstudie zur Wirkung von seriellen kalten, Kneipp'schen Obergüssen auf die Parameter der Lungenfunktion, die Anzahl der Infekte und die immunologischen Parameter von Patienten mit COPD wurde durchgeführt. Die Patienten hatten eine COPD mit einer FEV1 (forciertes exspiratorisches Volumen in der 1. Sekunde) $<80 \%$, eine stabile Erkrankung ohne Infektexazerbation in den letzten 4 Wochen, keine zusätzliche asthmoide Komponente, keine orale Kortikosteroidtherapie in den letzten 4 Wochen sowie keine schwerwiegende weitere Erkrankung. Das Alter der Patienten (3 w, $17 \mathrm{~m}$ ) betrug 64 Jahre (Median), die FEV1 lag im Mittel bei 63\% (Stadium II), die Erkrankungsdauer betrug 13,5 Jahre (Median). Der Studienablauf sah 3 Zeitabschnitte vor: der 1. Abschnitt (Baseline) begann zum Zeitpunkt des Einschlusses in die Studie (Kontrollzeitpunkt KP I) und erstreckte sich über 10 Wochen, danach begann nach KP II der 2. Abschnitt (Behandlungsserie), es wurden 10 Wochen $3 \times$ wöchentlich Kneipp'sche Obergüsse sowie $2 \times$ wöchentlich häuslich kalte Waschungen des Oberkörpers durchgeführt. Am Ende dieses Studienabschnittes folgte KP III. Die Nachbeobachtung (Follow-up) umfasste 12 Wochen und entspricht dem KP IV. Zu den einzelnen KP erfolgte die Bestimmung von Lungenfunktionsparametern (z.B. FEV1, Vitalkapazität) sowie PEF-Werten (forcierter exspiratorischer Fluss), von Blutgaswerten vor und nach Belastung (6-min-Gehtest), von Entzündungsparametern (CrP, BSG), Differentialblutbild, Zytokinexpression in Lymphozyten, Lymphozytensubpopulationen und das Erfassen des Befindens sowie der Lebensqualität. Die Anzahl der Infekte der oberen Luftwege jeweils in den letzten 10 bzw. 12 Wochen wurde registriert. Die Ergebnisse zeigten keine signifikante Beeinflussung der Lungenfunktionswerte durch Kneipp'sche Obergüsse. Jedoch zeigte der PEF, der jeweils vor und nach jedem einzelnen Oberguss gemessen wurde, jeweils einen signifikanten Anstieg um 4\% im Zeitraum der Hydrotherapie-Anwendung. Es war weiterhin ein teilweise signifikanter Anstieg des Blut-pH-Wertes im Studienverlauf zu beobachten, der auf einen gleichzeitigen Anstieg des Sauerstoffpartialdrucks $\left(\mathrm{pO}_{2}\right)$ und Abfall des Kohlendioxidpartialdrucks zurückzuführen war. Dies zeigt unserer Meinung nach eine Hyperventilation durch Rekrutierung vorher ungenutzter Lungenabschnitte durch den Kaltwasserreiz an. Die immunologischen Parameter machten deutliche Veränderungen sichtbar. So kam es zu einem signifikanten Anstieg der absoluten Lymphozytenzahl im Zeitraum der Serie um 13\%, diese fiel im Follow-up nicht auf den Ausgangswert von KP I zurück. Die Zytokinexpression der T-Lymphozyten machte einen klaren Trend deutlich: die der Th1-Reaktion (zellvermittelt) des spezifischen Immunsystems zugehörigen Zytokine (z.B. IFN- $\gamma$ ) stiegen im Verlauf der Wasserbehandlung an und fielen im Follow-up nicht auf ihre Ausgangswerte zurück, wohingegen die Zytokine der Th2-Reaktion (humoral) ab KP II kontinuierlich abfielen. Die Expression des Tumornekrosefaktors- $\alpha$ durch die Lymphozyten nahm im gesamten Studienverlauf kontinuierlich zu. Dies könnte die bei einer chronischen Entzündung vorhandene Unfähigkeit des Organismus, auf Reize adäquat zu reagieren, deutlich machen. Normalerweise reagiert ein Gesunder auf einen physikalischen Reiz mit der Abnahme von TNF- $\alpha$.

Auch eine signifikante Zunahme der Natural-Killer(NK)-Zellen (CD 16/56-CD3 ${ }^{-}$) konnte im Nachbeobachtungszeitraum gemessen werden. Dies zeigt möglicherweise die Aktivierung auch der unspezifischen Immunabwehr an. Die Lebensqualität zeigte in den verwendeten Fragebögen (SF-36 und St. Georges Respiratory Questionnaire, SGRQ) keine signifikanten Abweichungen. Das Patientenurteil wies eine Verbesserung des Befindens nach den seriellen, kalten Güssen bei $75 \%$ der Patienten auf, 25\% äußerten eine unveränderte Befindlichkeit. Die Anzahl der Infekte fiel im gesamten Studienzeitraum tendenziell ab, wobei der stärkste Abfall im Follow-up-Zeitraum zu verzeichnen war.

Zusammenfassend kann eine Abhärtung im Sinne einer funktionellen 
Adaptation immunologischer Parameter durch serielle Kaltwasserreize angenommen werden. Die komplexen Veränderungen der Immunparameter zeigen eine Modulation in Richtung des Th1-Typ-Musters (zelluläre Immunabwehr). Eine Abnahme der Infektanzahl bei COPD-Patienten durch kalte Kneipp'sche Obergüsse könnte möglich sein. Um solche Veränderungen im Sinne einer funktionellen Adaptation zu erreichen, sind jedoch große Zeitspannen von mehreren Monaten bzw. Jahren nötig.

\section{Literatur}

Bieger WP, Penz M, Gruber R: Immunologie der Abhärtungsreaktion nach Hydrotherapie. Phys Rehab Kur Med 1998;8:37-45,

Kreutzfeldt A, Albrecht B, Müller K: Einfluss des Wassertretens nach Kneipp auf die Immunregulation. Phys Med Rehab Kur Med 2003;13:208-214.

-Kroegel C: Die «Globale Initiative für chronisch-obstruktive Lungenerkrankungen» (GOLD). Aktualisierung der GOLD-Empfehlungen. Pneumologie 2004;58 65-68.

Werner GT, Drinovac V, Penz MG: Immunologische Untersuchungen zu Wirkungen der Kneipp'schen Hydrotherapie. Münch Med Wochenschr 1998;140:566-569.

\section{Atemtechniken als Weg zum Wohlsein}

\section{Dipl.-Sportl. Christine Irnich}

Universität München

Atemtechniken als Weg zum Wohlsein sind ein jahrtausendealtes Kulturgut der Menschheit. In Deutschland wurde in der 1. Hälfte des vorigen Jahrhunderts begonnen, wissenschaftlich den Atem zu erforschen. Inspiriert von Künstlern wie Clara Schlaffhorst (Sängerin), Hedwig Andersen (Pianistin), Cornelius Veening (Sänger) sowie von Frauen aus der «Reformgymnastik der 20er- und 30er-Jahre» des vorigen Jahrhunderts begann eine intensive Auseinandersetzung über die Funktion und Dimension des Atems für das menschliche Wohlbefinden.

Als erste Atemschule konsolidierte sich die Atem- und Stimmtherapie von Schlaffhorst-Andersen, die noch in der Weimarer Zeit «Rothenburger Schule» genannt wurde.

Die Pionierin Elsa Gindler gründete im Berlin der Weimarer Zeit eine Werkstatt zur praktischen Miterforschung des Atems und der Bewegung. Sie bildete Clara Fenichel, die Frau des körperorientierten Psychoanalytikers Otto Fenichel, Wilhelm Reichs spätere Lebensgefährtin Elsa Lin denberg und Erich Fromms Ehefrau als «Atemfrauen» aus. Alle namhaften Reformpädagogen waren eng mit Atemlehrerinnen befreundet. Aus dieser bewegungspädagogischen Arbeit Elsa Gindlers entwickelte sich später die Konzentrative Bewegungstherapie (KBT).

Der Arzt Johannes Ludwig Schmitt, bekannt als «Atemschmitt», verfasste 1948 sein über lange Jahre einzigartiges Buch «Atemheilkunst», erschienen in Bern 1948. Dieses Buch ist als Standardwerk über den Atem ein Konzentrat praktischer und theoretischer Arbeit. Es beschreibt die Atemmassage, den Weg zur Aktivierung von Atemreflexen und die Atembewegung als Zusammenspiel der Außeneinflüsse mit dem Vegetativum un der Seele. Durch seine Atemarbeit initiierte er eine «Wertungsänderung beim Patienten und Wandlung der Person in der Atemmassage.»

Sein Schüler Prof. Dr. med. Volkmar Glaser (Arzt und Atemlehrer) lernte bei ihm technisch exakt angesetzte Griffe. Deren Anwendung bewirkte bei Glaser jedoch wenig. In der Folge widmete er sich einer zentralen Frage, dem guten Kontakt zwischen Therapeut und Patient. Daraus ent wickelte er die so genannte Psychotonik, ein Atemverfahren zur Verbesserung der Kontakt- und Begegnungsfähigkeit unter Einbeziehung von Erfahrungen aus der asiatischen Heilkunde. Die Psychotonik basiert auf einem vital-sensorischen Miteinander. Wichtig ist dabei die Bereitschaftshaltung des Therapeuten, einen gemeinsamen sensorischen Raum anzubieten.

Eine wichtige, wenn nicht die wichtigste Pionierin der zweiten Generation auf dem Gebiet der Atemtherapie ist Ilse Middendorf, Schülerin von Elsa Gindler. Ilse Middendorf war Professorin an der Kunsthochschule für Musik und darstellende Kunst in Berlin und langjährige Leiterin der von ihr 1965 aufgebauten Ausbildungsstätte für Atemtherapeuten in Berlin, das heute «Ilse Middendorf Institut für den Erfahrbaren Atem» heißt und von Helge Langguth geleitet wird. Middendorf verfasste 1984 ein Standardwerk für Atemtherapeuten: «Der erfahrbare Atem». Ein Hauptaspekt dieses Buches ist die Dreiheit «Atmen, Sammeln, Empfinden». Sie postuliert: «Wir lassen den Atem kommen, wir lassen ihn gehen und warten, bis er von selbst wiederkommt.» Dies ist nur durch Gewahrwerden, durch Sammlung möglich. «Lassen» und «Zulassen» sind hier die ent- scheidenden Botschaften. Es kommt zu einem Loslassen verkrusteter Strukturen, es kommt zu einem Mehr an Gelassenheit. Bei Ilse Middendorf geht es um das Erfahren des Atems, nicht durch «Tun, sondern durch Lassen». Sie weist darauf hin, dass die Arbeit am Atem eine durchgehende Empfindungsfähigkeit verlangt. Die Empfindung breitet sich durch Atmen mehr oder weniger im Körper aus und wird räumlich-flächig erfahren. Erst danach antwortet das Gefühl mit all seinen Wertungen.

Auch C.G. Jung unterscheidet Fühlen und Empfinden als unterschiedliche Seelenfunktionen. Jede Art von Gefühl (Trauer, Freude, Wut usw.) drückt sich spezifisch im Atem, der Stimme, in der Bewegung, Gestik, Haltung, Mimik und der Herzfrequenz aus. Dieser bestimmte Ausdruck ist universell, d.h. in allen Kulturen gleich anzutreffen.

Der Psychoanalytiker Wilhelm Reich entdeckte als einer der Ersten an seinen Patienten, dass sie emotionale, körperliche Reaktionen und $\mathrm{Ge}-$ fühle durch dauernde muskuläre Spannungen kontrollieren und binden. Die Patienten hemmen die Atmung. Das Ergebnis ist ein Affektverlust und eine Herabsetzung der emotionalen Spannung sowie eine Einschränkung der Bewegungsfähigkeit des Körpers.

Eine der einfachsten Möglichkeiten, den Atem zu beeinflussen und aus festgelegten und einschränkenden Mustern zu befreien, bietet Bewegung. Bewegung beeinflusst den Atem, ohne dass wir willentlich eingreifen müssen. Viele körperorientierte Verfahren bedienen sich dieser Erkenntnis. Sie nutzen Bewegung und beeinflussen damit immer auch die Atmung. Es gibt zahlreiche Atemtechniken aus unterschiedlichen Kulturen. Sie haben in vielen westlichen körperorientierten Verfahren einen hohen Stellenwert. Neben der klassischen Atemtherapie wird der Atmung v.a. in der Psychotonik nach Glaser, der Rhythmik, der KBT, Feldenkrais, aber auch im Sport (Gymnstik, Ausdauersportarten usw.) ein großer Wert beigemessen und ist Grundbestandteil dieser Therapien. Moshe Feldenkrais sagte: «Gute Atmung bedeutet gute Haltung, ebenso, wie gute Haltung auch gute Atmung bedeutet.»

Östliche Atemtechniken finden sich im Qigong, Yoga, den Kampfkünsten (Wushu, Taek Won Do, TaiChi usw.) und der Meditation wieder. Yoga (die Schwester des Qigong) kennt Übungen wie Meditation, Askese, Pranayama (Atem-Übungen), Nidra (Tiefenentspannung), Asanas (Körperhaltungen), Nadi Shodhana (Wechselatmung). Qigong basiert auf langsamen, bewussten Bewegungen unter Einbeziehung der Vorstellungskraft im Sinne der TCM und führt dadurch zu einer tiefen, ruhigen Bauchatmung. Durch regelmäßige Qigong-Übungen kommt es zu einer Beruhigung des Sympathikotonus, zu einer Stimmungsaufhellung und inneren Haltungsänderung. Dies wiederum wirkt sich positiv auf die Atmung aus. Auch bei der Zen-Meditation wird der Atembewegung Aufmerksamkeit und Achtsamkeit geschenkt. Auch hier, obwohl äußerlich nicht sichtbar, geht es um Atem und Bewegung, innere Bewegung.

All diese Methoden bedienen sich der Bewegung zur Entspannung, Bewusstwerdung, Regulierung der Atmung, Haltungsänderung (innere und äußere), Harmonisierung und Eutonisierung. Sie haben ihre z.T. jahrtausendealte Wurzel im ganzheitlichen Ansatz von Körper, Geist und Seele. Ihnen gemeinsam ist eine konzentrierte Ruhe, eine langsame Bewegung und Achtsamkeit im Moment. Sie basieren darauf, die «Horde wilder Affen» im Gehirn zu bändigen (keep the monkeys calm) und über sanfte Bewegung zu einer Harmonisierung, Erdung und Präsenz zu gelangen. Dadurch wird der Atem sich unwillkürlich in eine tiefe, gelassene, bis in tiefe Schichten sich ausbreitende Atmung verwandeln.

Ziele dieser Atemtechniken sind Haltungsänderungen, Lösen von Ver krustungen, das Schaffen von Ressourcen, körperliche und geistige Stärkung, Eutonisierung (Wohlspannung) und das Aktivieren von Selbstheilungskräften. Ziel- und Zweckorientiertheit sind hier fehl am Platz, in der Arbeit mit dem Atem geht es um Empfindungsfähigkeit. Nur so kann es zu einer tiefen und grundlegenden Haltungsänderung und zu einer Aktivierung der eigenen Ressourcen kommen. Diese Aktivierung kann dann zur Selbstheilung und zu dauerhaftem Wohlsein führen.

\section{Literatur}

Schmitt J-L: Atemheilkunst, ed 5. Bern, Humata,1977.

Middendorf I: Der erfahrbare Atem. Eine Atemlehre, ed 8. Paderborn, Junfermann, 2001

Volkmar G: Eutonie. Das Verhaltensmuster des menschlichen Wohlbefindens. Lehrund Übungsbuch für Psychotonik, ed 4. Heidelberg, Haug, 1993.

Sakyong Mipham: Wie der weite Raum. Die Kraft der Meditation. München, dtv, 2005 .

Guorui J: Qigong Yangsheng. Uelzen, MLV, 1998.

Hildenbrand G: Qigong - chinesische Heilkunde in Aktion. Akupunktur 1999;27: $76-85$

Patañjali: Die Wurzeln des Yoga: Die klassischen Lehrsprüche des Patañjali - die Grundlage aller Yoga-Systeme. Frankfurt, O.W. Barth, 2003

Trökes A: Das große Yogabuch, ed 2. München, GU, 2001. 